\title{
POST-ENCEPHALITIC PARKINSONISM WITH AMYOTROPHY
}

\author{
BY \\ J. G. GREENFIELD and W. B. MATTHEWS \\ From the Neurological Department, King's College Hospital, and the Institute of Neurology, \\ Queen Square, London
}

The syndromes of amyotrophic lateral sclerosis and progressive spinal muscular atrophy are regarded by many authors as differing in some respects from the group of systematized degenerations of the nervous system (Wilson, 1940). One reason for this segregation is that apparently systematized lesions, producing a similar clinical condition, may on occasion be produced by a variety of aetiological agents. Among these agents the virus of epidemic encephalitis has frequently been incriminated but has been largely overlooked by British authors. It therefore seems worth while to report on two patients in whom " motor neuron disease" was superimposed on Parkinsonism which was attributable to encephalitis, with certainty in one case and with strong probability in the other.

Case 1. J.C. 17694. - A man aged 39 was first admitted to King's College Hospital under Dr. S. Nevin in November, 1948. At the age of 18 he had had an attack of " influenza" during which he was unconscious for about two weeks and it seems very probable that this was an acute episode of encephalitis although no further details are available. He remained well until the age of 28 when he developed slowly increasing tremor of the right hand and stiffness and slowness of movement of the right arm, soon spreading to involve the left arm. At about this time he began to hold his head tilted to the left and this persisted until his admission. Aged 31 he began to have oculogyric crises in which his eyes would turn upwards and remain there for several hours during which time he would have compulsive thoughts about his financial worries. He first attended hospital with these symptoms at the age of 36 and was thought to show the typical appearances of post-encephalitic Parkinsonism and eventually he was admitted for a trial of the newer forms of treatment.

The only possibly significant past history was of an attack of "St. Vitus dance" at the age of 12 , and no neurological disease was known in the family.

On his admission in 1948 he showed the features of a moderately severe post-encephalitic Parkinsonism with tremor of both hands, rigidity of all limbs, immobile facies, blepharoclonus and, in addition, flexion of the neck to the left. Oculogyric crises, which were occurring about once a week, were observed in hospital. All the tendon reflexes were very brisk but the plantars were flexor. No wasting was seen but fasciculation was observed in the upper arms.

His tremor was greatly relieved by treatment but the oculogyric crises continued unabated. In 1949 he first noticed weakness of the right arm, but paid little attention to this until 1952, when wasting of the right hand became evident and on this account he was again admitted to hospital in September, 1952.

On examination he was intelligent and had good insight into his condition. There was no anosmia and the optic fundi and vision were normal. The external ocular movements were full, apart from inability to converge, and the pupils reacted normally to light but not on attempted convergence. The facies had a masklike appearance and there was well marked blepharoclonus but there was no weakness of the facial muscles. There was no sensory loss on the face and hearing was normal. The palate and tongue were normal in appearance but his speech was unduly rapid and monotonous in tone. His head was held slightly flexed to the left and could only be held upright by an effort of concentration.

Obvious wasting was present in the muscles of the right hand and forearm and to a lesser extent on the left, with weakness of similar distribution. Fasciculation was widespread in the muscles of both hands and arms and of the shoulder girdle. No weakness, wasting, or fasciculation was observed in the legs. A moderate degree of rigidity of cogwheel type was present in all limbs and both hands showed an intermittent Parkinsonian tremor. The tendon reflexes were all brisk, the left plantar was flexor and the right probably extensor. No sensory loss was found.

The cerebrospinal fluid was under normal pressure with no evidence of spinal block and contained 1 cell per c. $\mathrm{mm}$. and $15 \mathrm{mg}$. of protein per $100 \mathrm{ml}$. The Wassermann reaction was negative in the blood and cerebrospinal fluid and radiographs of the cervical spine were normal. Partial reaction of degeneration was present in the small muscles of the right hand and electromyography with needle electrodes showed fasciculation and fibrillation potentials in the same muscles.

When last seen on March 18, 1953, the wasting and weakness had advanced in both arms but the tremor was now less pronounced and was confined to the right hand. 
The tendon reflexes in the arms were now definitely exaggerated and both plantars were now extensor.

This patient developed Parkinsonism, torticollis, and oculogyric crises 10 years after a presumed attack of encephalitis, and, after a further 12 years, a condition clinically indistinguishable from amyotrophic lateral sclerosis.

Case 2. F.W.*-A man aged 57 was admitted to King's College Hospital in July 1952 under Dr. MacDonald Critchley. At the age of 31 he first experienced slight difficulty in walking due to a feeling of. stiffness beginning in the left foot and soon spreading to the right. This was attributed to flat feet and, although his abnormal gait was noticeable to others, the disability was slight and he did not consult a doctor. At the age of 42 he first developed a tremor of the left hand and it was noticed that he did not swing this arm when walking. The tremor soon involved the right arm and was accompanied by slowness of movement and fatigue on slight exertion. The gradual advance of these symptoms made his office work and travelling increasingly burdensome, and in 1948 he first noticed that, when he returned exhausted in the evening, his eyes would become fixed in gaze to the right. This could be overcome by a voluntary effort, but the eyes would revert to their former position when his concentration was relaxed and would only return to normal after he had rested for an hour.

At about this time he was recognized as a case of Parkinsonism but derived little benefit from treatment except that the ocular symptoms were seldom troublesome after 1950. After walking a short distance he would develop an acute flexion of the trunk and was beginning to have difficulty in turning over in bed and in getting into his bath. In September, 1951, he noticed a curious sensation of discomfort and distension in all the muscles of the trunk and limbs. He thought that his limbs were becoming generally thinner and he was having increased difficulty in walking due to weakness rather than to stiffness of the legs. In October, 1951, he first attended the neurological clinic at King's College Hospital.

At that time he showed the signs of moderately advanced Parkinsonism with immobile facies, rigidity of all limbs, and tremor of both hands, the left more than the right. His posture was not the typical attitude of flexion but the spine was held extended with a marked lumbar lordosis. There was no disturbance of reflexes and, although the limbs were thin, there was no localized wasting and no fasciculation.

In spite of treatment his symptoms progressed so that by December, 1951, he could no longer dress or shave himself or get out of bed unaided. He felt an increasing general weakness and, in January, 1952, complained of specific weakness of the right arm. The reflexes in the arms were now diminished and fasciculation was seen in many muscles in all limbs but no reaction of degeneration was found in the muscles of the right arm. Tremor and

\footnotetext{
${ }^{*}$ This case is that referred to as No. 31 in the article on "The Brain-stem Lesions in Parkinsonism" by J. G. Greenfield and F. D. Bosanquet. 1953. Journal of Neurology, Neurosurgery and Psychiatry, 16, 213.
}

rigidity were still marked in the arms. In March, 1952, there was obvious localized wasting of the muscles of the right hand, and electromyography showed fibrillation potentials in these muscles. The patient was reluctant to enter hospital but increasing weakness and alarming symptoms of breathlessness at rest and regurgitation of fluids down the nose finally led to his admission in July, 1952.

No history of a possible attack of encephalitis in the past could be obtained and no similar disease had occurred in the family.

On admission he was almost helpless and lay motionless in bed and was unable to feed himself. His mental condition appeared normal. There was no anosmia and the optic fundi and pupils were normal. The external ocular movements were full apart from restricted convergence ; there was no sensory loss on the face and no obvious weakness of the face or jaw. The palate moved centrally but through a small range and movements of the tongue were slow although there was no wasting.

The entire musculature of the trunk and limbs was severely and symmetrically weak and wasted, the proximal muscles of the arms, in particular, being almost powerless and the chest expansion negligible. Constant fasciculation was present in the muscles of the arms and shoulder girdle. Tremor was now only slight and intermittent and rigidity, while still present, was less than formerly. The tendon reflexes were absent in the arms but were present and normal in the legs and the plantars were flexor. There was no sensory loss.

The blood pressure was $150 / 95 \mathrm{~mm}$. $\mathrm{Hg}$ and, apart from rhonchi at the bases of both lungs, no abnormality was found on general examination.

The cerebrospinal fluid was under normal pressure with no evidence of spinal block and contained 2 cells per c. mm. and $35 \mathrm{mg}$. of protein per $100 \mathrm{ml}$. The Wassermann reaction was negative in the blood and cerebrospinal fluid.

In the succeeding few days he had frequent choking attacks while eating. Respiration became increasingly embarrassed and on July 7 he had repeated attacks of respiratory arrest with loss of consciousness and died that night. A partial necropsy was performed 36 hours after death, the brain and spinal cord being removed and fixed in formol saline.

\section{Histology of Brain and Spinal Cord}

Large celloidin blocks were prepared from the frontal, Rolandic, parietal, and occipital lobes, from the basal ganglia at five levels in the coronal plane, and from the cerebellum. Sections from these were stained by thionin, haematoxylin-van Gieson, haematoxylin phloxin-tartrazin (Lendrum), and Mallory's phosphotungstic acid haematoxylin. In addition frozen sections from the frontal, Rolandic, hippocampal, and temporal regions of the cortex were stained by the silver methods of van Braunmühl and Bielschowsky. The mid-brain, pons, and upper medulla were embedded in paraffin 
after being mordanted for several days in saturated mercuric chloride solution, and sections from them were stained by thionin, Lendrum's method (as above), haematoxylin-van Gieson, phosphotungstic acid haematoxylin, Azan, Congo-red, and Holmes' reduced silver method. Serial sections were made of the mid-brain. Sections from various segments of the cervical, thoracic and lumbo-sacral regions of the spinal cord were made after embedding in paraffin or celloidin. These were stained by thionin, Lendrum's method, haematoxylin-van Gieson, Azan, Loyez myelin method, phosphotungstic acid haematoxylin, and the Gros silver method. Sections from two levels of the lumbo-sacral enlargement were also stained by the Swank-Davenport method for degenerating myelin.

Cortex.-Sections of the cortex show no abnormality. In sections stained by silver impregnation no senile plaques or nerve cells showing Alzheimer tangles can be seen either in the hippocampus or isocortex.

Caudate-putamen and Globus Pallidus.-In the basal ganglia the caudate-putamen (corpus striatum) appears quite normal. In the globus pallidus there is a diffuse and definite loss of nerve cells, and excess of lipochrome in many that remain. There is also a slight diffuse gliosis, in the sense that sparse neuroglial fibres can be seen running long distances across many high-power fields, but spider cells are not seen. The larger arteries show incrustation of their media, and there is a narrow but dense layer of neuroglial fibres around them.

No changes can be seen in the corpus Luysii.

Substantia Nigra.-In the substantia nigra there is a widespread and severe loss of nerve cells with considerable glial scarring in the groups in which the greatest cell loss has occurred. The best preserved groups are the most mesial, that is, those nearest the sulcus oculomotorius.

Many cells, especially in the oral and lateral parts of this nucleus, show neurofibrillary change which appears as a faintly haematoxophilic, highly refractile striation, with loss of Nissl granules. In some cells it affects only one half, but in most cases the whole of the nerve cell is involved. Melanin pigment has disappeared from such cells in part or entirely. Sometimes scattered melanin granules in or round the margin of the cell draw attention to its presence, especially in sections stained by thionin, which leaves the cell body unstained. Some cells appear to retain their limiting membranes, and appear rounded, pyriform, or bipolar, but others appear as tangles of coarse fibrils, of the same size or rather larger than the neighbouring nerve cells, and more or less rounded or triangular in shape ; they appear to have lost their nucleus and their limiting membrane. With Congo red and to a less extent with Lendrum's stain these tangles appear anisotropic, but not brilliantly so.

Two or more such degenerated nerve cells may be seen fairly close together, but in the substantia nigra many fields, and in fact many serial sections, may be searched without any being found; in the locus coeruleus they were more abundant.

Throughout the substantia nigra there is a tendency for the nerve cells to contain more lipochrome pigment than normal. This usually lies, as a rounded, colourless or faintly yellow mass of granules, between the nucleus and the melanin pigment.

A binucleated nerve cell is present in the mid-zone of the caudal part of the substantia nigra. Occasional cells contain single vacuoles, which may be as large as the nucleus, and which usually lie under the margin of the cell. The Nissl granules in these cells stain well, though they and the nucleus may be displaced. Careful examination shows that these vacuoles are in fact empty of any stainable cytoplasm. They are seen not only in sections stained by thionin but also in those stained by more complete methods.

In the lower part of the oculomotor nucleus two cells are seen containing single concentric Lewy inclusions with a sharply defined phloxino-philic core (Figs. 1 and 2). Several cells in this nucleus on both sides of the mid-line are undergoing neurofibrillary degeneration (Figs. 3 and 4) and a senile plaque was found in this nucleus in a section stained by Holmes' method (Fig. 5). A few of the pigmented cells of the locus coeruleus also show neurofibrillary change in varying degree, sometimes so severe that the cell appears merely as an abnormal orientation in the fibrillar structure of the ground substance. Anisotropism with Congo red and with Lendrum's stain show these to be true neurofibrillary tangles.

The medulla shows no abnormality except an excess of lipochrome in the cells of the vestibular nuclei. No gross abnormality was seen in the nucleus ambiguus or hypoglossal nucleus.

Spinal Cord.-In the cervical enlargement (5-8C) there is a general loss of nerve cells, which appears greatest in the fifth and sixth segments. Many nerve cells are shrunken and some contain a great excess of lipochrome. In a few a more homogeneous rounded area is seen among the lipochrome granules, but no typical Lewy inclusions. Various degenerative changes are present in some of the cells. Occasional hyaline acidophilic and argyrophilic 


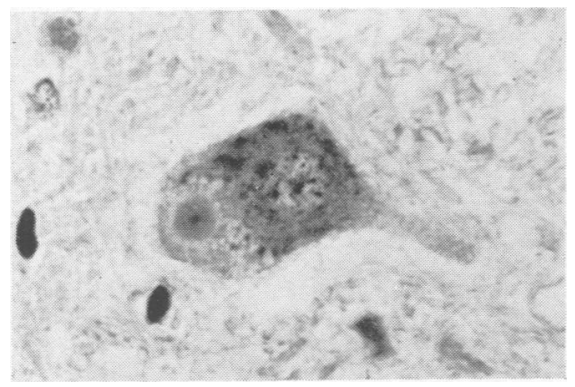

Fig. 1

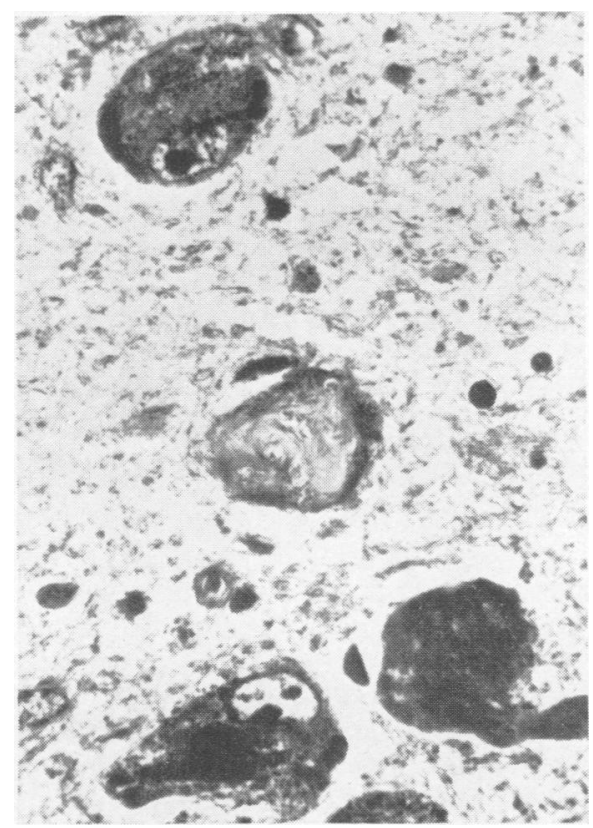

FIG. 3

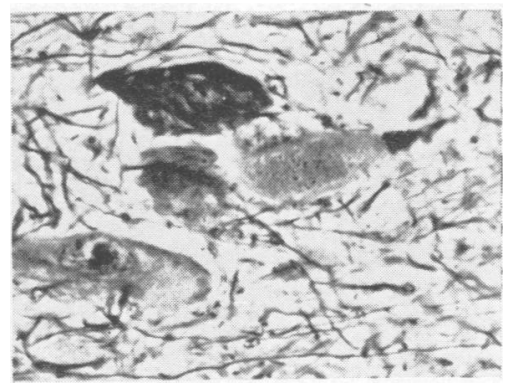

Fig. 4

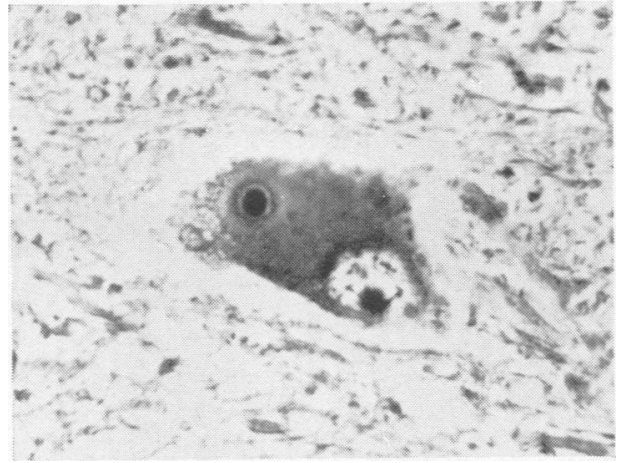

FiG. 2

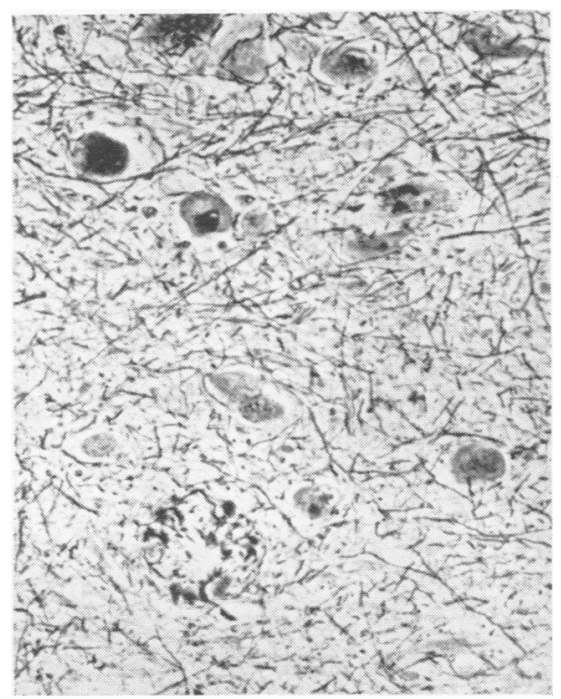

FIG. 5

FIG. 1.-Concentric cytoplasmic inclusion of Lewy type in a cell of the oculomotor nucleus. Lendrum's phloxin tartrazine. $\times \mathbf{6 6 0}$.

FIG. 2.-Concentric Lewy inclusion in cell of oculomotor nucleus. Lendrum's phloxin-tartrazine. $\times 900$.

FIG. 3.-Neurofibrillary change in cell of oculomotor nucleus. Haematoxylin-van Gieson. $\times 600$.

Fig. 4.-Neurofibrillary change in cell of oculomotor nucleus. Holmes' reduced silver method. $\times 450$.

Fig. 5.-Senile plaque in oculomotor nucleus. Holmes' reduced silver method. $\times 200$. 


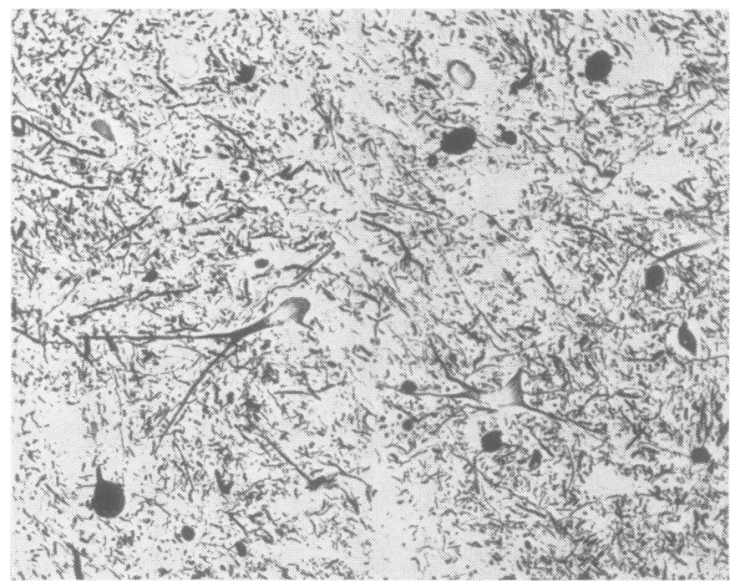

Fig. 6.-Axonal swellings in the base of the ventral horn of the eighth cervical segment. Gros silver technique. $\times 100$.

bodies are seen lying among or just dorsal to the nerve cells in the ventral horn; some reach the size of the smaller nerve cells (Fig. 6) ; they appear to be swollen axons. In the sixth thoracic segment the ventral horn cells appear shrunken, though not much reduced in number ; many contain an excess of lipochrome, which may push the nucleus against the cell margin.

In the lumbo-sacral enlargement the cell loss is very obvious, especially in the lateral group of the ventral horn, where very few nerve cells remain.

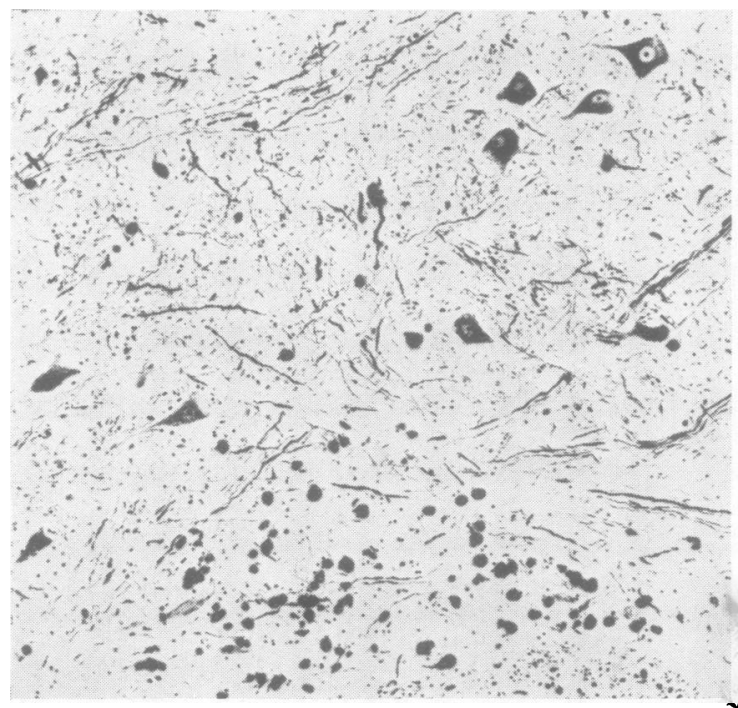

FIG. 7.-Argyrophilic balls (possibly axonal swellings) in the antering border of the ventral horn. Lumbar segment. Gros si' technique. $\times 100$.
Many of these are small bodies consisting chiefly of lipochrome granules among which are sparse Nissl granules. The few larger nerve cells which remain also contain an excess of lipochrome. Swellings on the axons are very noticeable here. A few large swellings about half the diameter of the larger nerve cells are seen internal to the postero-lateral group of nerve cells. On the anterior border of the ventral horn there are numerous smaller argyrophilic bodies, which may form clumps of 20 or more (Fig. 7). These seem to be common findings in the lumbar cord (Dr. Marion C. Smith, personal communication). Many of the axons near the surface of the cord in all the columns are somewhat swollen, but no tract degeneration could be seen by Loyez myelin stain. There is considerable neuroglial overgrowth in the grey matter in the more affected segments of the cervical and lumbo-sacral enlargements. It is greatest at the bases of both ventral and dorsal horns where the branches of the anterior spinal artery are surrounded by zones of fairly dense fibrous gliosis. Elsewhere, especially where the loss of nerve cells is greatest, many binucleated and some trinucleated astrocytes, with thicker processes than normal, are present.

The sections stained by osmic acid showed no abnormality except excess of lipochrome in the nerve cells.

\section{Discussion}

A variety of forms of amyotrophy has been attributed to epidemic encephalitis. Many of these have been localized, radicular, and non-progressive and usually evident immediately after the acute attack (Margulis, 1926). In a further group of cases the clinical picture has not resembled motor neuron disease, the wasting being hemiplegic, (Roger and Cain, 1947 ; Decourt, Mathieu, and Meyer, 1934 ; Grinker, 1920), remittent (Froment and Gennevois, 1921 ; Froment, 1925), or accompanied by sensory loss (Roger and Cain, 1947). There remain, however, a number of case reports of a clinical syndrome closely resembling motor neuron disease coexisting with undoubted evidence of chronic encephalitis.

The frequency with which this combination has been reported makes it unlikely that this is a mere chance association of two diseases. The most important contribution is that of Wimmer and Weel (1928) who reported 20 cases, and, while it is difficult to accept all their case reports, they seem is have established that a progressive, apparently matized, spinal amyotrophy, with or without midal involvement, may occur as part of a encephalitic syndrome. The clinical features 
may be illustrated from the reports of other authors. Amyotrophy may develop immediately after the acute attack of encephalitis (Jancsó, 1928), after a brief delay (Ornsteen, 1930), or after a more prolonged interval, e.g. seven years (Carr, 1926; Illing, 1932 ; Lhermitte, Thibault, and Ajuriaguerra, 1938), 10 years (Kral, 1938), or even 18 years (Milhorat, 1946). The muscles of the arms are usually affected first but occasionally bulbar palsy may be the presenting symptom (Jancsó, 1928). Signs of pyramidal disease were present in most of the reported cases, but may be absent throughout (Milhorat, 1946) as in the present Case 2. Progression may be rapid but many patients survive longer than is usual in amyotrophic lateral sclerosis.

In many of the cases reported shortly after the years of the epidemic the history of encephalitis was indisputable. Acceptable signs of a postencephalitic syndrome are often present such as continued sleep disturbance (Salus, 1929), obesity and narcolepsy (Kral, 1938), and oculomotor disorders (Wimmer and Neel, 1928). Parkinsonism was regarded as rare by Wimmer and Neel, only one of their cases showing the full syndrome, although two others showed minor Parkinsonian features. Parkinsonism was present in the cases reported by Illing (1932), Lhermitte and others (1938), Milhorat (1946), and Roger and Cain (1947), and oculogyric crises in those of the two former authors.

Pathological examinations have been infrequent. Wimmer and Neel (1928) reported the findings in two cases. In their Case 3 the original attack of encephalitis lethargica appears to have occurred in 1925 when the patient, aged 47, came into hospital in a delirious, somnolent state with hyperthermia, associated with bilateral ptosis, inequality of the pupils, tremor of slow, coarse, irregular character in the right arm and myoclonic jerkings of the shoulders, arms, back, and legs. On recovery there was weakness of lateral movements of the eyes, and atrophy appeared in the masseters, temporals, and hand muscles and also, though to a slighter degree, in the muscles of the arms and shoulder girdle. An extensor plantar response was noted on the left side. Parkinsonism came on rather later but was evident in 1926, the year in which he died. On post-mortem examination there were gross changes in the neurons of the ventral horns of the cervical spinal cord, with considerable loss of these cells. Cuffing with lymphocytis and plasma cells was seen round many vessels. There was pallor of the pyramidal and antero-lateral tracts in the cervical cord, but the lumbar cord was almost normal. Their Case 16 was that of a man of 36 who had an acute attack of encephalitis lethargica in 1919. By December of the same year there was increasing weakness of the left hand with muscular atrophy. Weakness of the left leg and increasing rigidity of all the limbs and of the body came on soon after, with dysphagia and spasmodic laughter and crying. In April, 1921, there was great rigidity of the whole body, increase of tendon reflexes and extensor type of plantar response, atrophy of the arms and myoclonus of the face and limbs. Postmortem examination was limited to the brain, and here also perivascular infiltration was seen.

Lhermitte and others (1938) published a case in which Parkinsonism followed five years, and amyotrophy seven years, after a typical attack of encephalitis lethargica. At this stage lateral oculogyric cases were observed, along with myoclonus and tonic spasms in the right hand. The amyotrophy spread to involve most of the muscles of the hands, arms, and shoulder girdles, with fibrillations and fasciculations and asynchronous myoclonus. There was increase in deep reflexes and positive Oppenheim reflexes. Later weakness of masseters, tongue, and soft palate caused severe dysphagia which led to death 13 years after the onset of the disease. Post-mortem examination of the spinal cord showed the greatest change in the cervical and upper thoracic segments where there was pallor of all the marginal zone with incomplete degeneration of the spino-cerebellar tracts and slight degeneration of the pyramidal tracts and dorsal columns. The ventral horn cells were reduced in size and number, with some ghost cells. No inflammatory changes were noted.

In our Case 2 the interval between the original attack of encephalitis, which is unlikely to have occurred after 1926, and the onset of amyotrophy was considerably greater, more than 20 years. There were no signs of pyramidal involvement and no degeneration was found in the long tracts of the spinal cord. The absence of perivascular infiltrations may be related to the long interval between the original attack and death, and agrees in this respect with the findings in the mid-brain and basal ganglia in post-encephalitic Parkinsonism. Otherwise our case conforms to the clinico-pathological picture presented by the other cases. It is noteworthy how frequently myoclonus has been observed in the limb muscles in cases going on later to amyotrophy, suggesting a direct attack by the virus on the anterior horns of the spinal cord. The problem of the pathogenesis of post-encephalitic amyotrophy is the same as that of post-encephalitic Parkinsonism. It may be due to premature degeneration or abiotrophy affecting neurons which have been attacked by the virus in the acute phase of the 
disease, or it may reflect the continued action of the virus in a modified form, as was held by von Economo (1931) and most French writers. The presence of inflammatory infiltrations in the earlier years after the original attack is in favour of the latter hypothesis. Von Braunmühl (1949) considers neurofibrillary tangles, which are commonly found in the pigmented cells of the brain stem in post-encephalitic Parkinsonism (Greenfield and Bosanquet, 1953) as evidence of a prolonged toxic action, presumably by the virus of the disease. The finding of similar changes in the cortical neurons in cases of subacute inclusion or sclerosing encephalitis, which have survived for several years (Malamud, Haymaker, and Pinkerton, 1950 ; Corsellis, 1951), supports this view.

Just as Parkinsonism may appear immediately after an attack of encephalitis lethargica, or after an interval of a few or many years, so also with amyotrophy. In Milhorat's Case 1 the first symptoms both of Parkinsonism and amyotrophy appeared after the unusually long interval of 18 years. In our cases the interval was shorter for Parkinsonism but probably longer for amyotrophy (more than 20 years in both cases).

In our Case 2 a close correlation could be seen between the clinical picture and the post-mortem findings. The Parkinsonism could be related to severe loss of cells in the substantia nigra and locus coeruleus, and degenerative changes in the globus pallidus. The oculogyric crises may possibly be related to changes in the oculomotor nuclei, where not only neurofibrillary degeneration, but also Lewy inclusions and a senile plaque were found. Lewy inclusions were seen by Lafora (1913) in the oculomotor nucleus in cases of Parkinsonism ; no clinical details were given of these cases, but the date indicates that they were probably not postencephalitic. Changes in the oculomotor nucleus were also seen by Hallervorden (1933) in postencephalitic cases; but most workers on the sequelae of encephalitis lethargica have paid little attention to this nucleus in spite of its clinical involvement in the original disease. Some tend to relate oculogyric crises rather to disturbances in the vestibular nuclei, although changes in them have rarely been described.

The relation of amyotrophy to loss of anterior horn cells was evident in our case, as in the other post-mortem cases. No changes characteristic of post-encephalitis, such as neuro-fibrillary changes, were seen in the spinal cord, but the presence of numerous large axonal swellings in the bases of the ventral and dorsal horns was an unusual finding.

\section{Summary}

A description is given of two cases of postencephalitic Parkinsonism with oculogyric crises, both of which developed progressive spinal amyotrophy many years after the onset of Parkinsonism. One patient died from dysphagia and respiratory embarrassment. A full examination of the brain and spinal cord showed degeneration in the globus pallidus, the pigmented cells of the mid-brain and pons, oculomotor nuclei, and anterior horns of the cervical and dorsal enlargements of the spinal cord. There were no tract degenerations.

The pathogenesis of post-encephalitic amyotrophy is discussed.

We are grateful to Drs. Macdonald Critchley and S. Nevin for permission to use the clinical notes of these cases.

\section{REFERENCES}

Braunmühl, A. von (1949). Arch. Psychiat. Nervenkr., 181, 543.

Carr, A. D. (1926). Arch. Neurol. Psychiat., Chicago, 16, 344.

Corsellis, J. A. N. (1951). J. ment. Sci., 97, 570. 41 (2), 596.

Economo, C. von (1931). Encephalitis Lethargica, trans. K. Newman. Oxford University Press, London.

\begin{tabular}{l} 
Froment, J. (1925). Rev. neurol., Paris, 32 (1), 842. \\
\hline , and Gennevois, E. (1921). Ibid., 28. 726.
\end{tabular}

Greenfield, J. G., and Bosanquet, F. D. (1953). Journal of Neurology, Neurosurgery and Psychiatry, 16, 213.

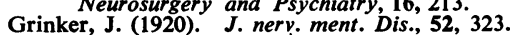

Grinker, J. (1920). J. nerv. ment. Dis., 52, 323.
Hallervorden, J. (1933). Klin.Wschr., 12, 692.

Illing, E. (1932). Mschr. Psychiat. Neurol., 82, 177.

Jancsó, S. (1928). Klin. Wschr., 7, 2442.

Kral, A. (1938). Mschr. Psychiat. Neurol., 98, 340.

Lafora, G. R. (1913). Trab. Lab. Invest. biol. Univ. Madr., 11, 29. Lhermitte, L., Thibault, and Ajuriaguerra (1938). Rev. neurol., Paris,

69, 372 . J. Path., 26, 133.

Margulis, M. S. (1926). Dtsch. Z. Nervenheilk., 89, 262.

Milhorat, A. T. (1946). Arch. Neurol. Psychiat., Chicago, 55, 134. Ornsteen, A. M. (1930). J. nerv. ment. Dis., 72, 369.

Roger, H., and Cain, J. (1947). Bull. Acad. nat. Méd., Paris, 131, 461. Salus, F. (1929). Dtsch. Z. Nervenheilk, 109. 259.

Wilson, S. A. Kinnier (1940). Neurology. Arnold, London.

Wimmer, A., and Neel, A. V. (1928). Acta Psychiat., Kbn., 3, 319. 